\title{
INNER PRODUCTS IN COVOLUME AND MIMETIC METHODS
}

\author{
KATHRYN A. TRAPP ${ }^{1}$
}

\begin{abstract}
A class of compatible spatial discretizations for solving partial differential equations is presented. A discrete exact sequence framework is developed to classify these methods which include the mimetic and the covolume methods as well as certain low-order finite element methods. This construction ensures discrete analogs of the differential operators that satisfy the identities and theorems of vector calculus, in particular a Helmholtz decomposition theorem for the discrete function spaces. This paper demonstrates that these methods differ only in their choice of discrete inner product. Finally, certain uniqueness results for the covolume inner product are shown.
\end{abstract}

Mathematics Subject Classification. 65N06.

Received March 26, 2007. Revised February 29, 2008.

Published online July 30, 2008.

\section{INTRODUCTION}

This paper presents a class of compatible spatial discretization methods for solving partial differential equations. This class includes the mimetic numerical method of Hyman and Shashkov which relies on primary and dual discrete differential operators and the covolume method of Nicolaides, which relies on a dual mesh, in two dimensions a Delaunay-Voronoi triangulation. Both of these methods have been successfully applied to problems in electromagnetics, heat, diffusion and fluid flow $[2,9,13,15,16]$. We demonstrate that these two methods differ only in the choice of discrete inner products on the discrete function spaces and in this way we extend these methods to an entire class of compatible discretizations.

In the equations of electromagnetics, discretizations that do not preserve fundamental vector calculus properties may lead to spurious approximations. For example, if the divergence-free condition on the magnetic field is not preserved in an appropriate sense at each time step physically unrealistic solutions can result. For this reason, recent attention has been paid to the importance of compatible spatial discretizations; methods which appropriately preserve important physical relations expressed by vector calculus in the continuous system of differential equations.

In the application of finite element methods to Maxwell's equations it is the edge elements of Raviart-Thomas and Nedelec $[11,12,17]$ that preserve the necessary conforming properties. That these function spaces preserve composition properties, integral identities, and existence of potential function properties is most easily seen through the use of exact sequences. Arnold has elegantly described these discrete differential complexes and the connection between edge elements and Whitney forms has been explored by Bossavit and Hiptmair $[4,5]$.

Keywords and phrases. Compatible discretization, discrete Helmholtz orthogonality, discrete exact sequence, mimetic method, covolume method.

1 University of Richmond, Richmond, VA, USA. ktrapp@richmond.edu 
These sequences have been used to analyze the stability of certain function spaces for other partial differential equations [1].

The discretization algorithms in this paper may be viewed as generalized finite difference techniques: they serve as a useful and often simpler alternative to the finite element method. Using discrete differential operators they provide a direct discretization of systems of partial differential equations from electromagnetics, diffusion and fluid flow. As they do not rely on variational forms of the equations they are particularly amenable to solving the full time-varying set of Maxwell's equations.

The covolume method of Nicolaides and the mimetic method of Hyman and Shashkov were both developed in the 1990s and by design preserve standard vector calculus properties. In addition to providing physically realistic approximations, the discrete vector calculus structure on which these methods are based is an essential part of the convergence rate analysis. In particular, the discrete Helmholtz decomposition is crucial in demonstrating existence and uniqueness of solutions $[10,13,18]$. A discrete exterior calculus (DEC) has also recently been studied by Hirani for more general discrete manifolds, and here the focus is on preserving the general Stokes' Theorem and a Hodge decomposition [6].

This paper places the mimetic and covolume methods within a discrete exact sequence framework from which it becomes clear that they belong to an entire class of algorithms that preserve essential calculus identities. This structure guarantees discrete analogs of Stokes' Theorem and a Helmholtz decomposition among other essential vector calculus properties.

This framework allows for new insights into the covolume method by viewing it as a dual operator method. It allows for convergence analysis from the covolume method to be adapted to prove convergence for the mimetic method in certain systems of equations [18]. Finally, this view lends itself to developments in higher-order difference methods.

In the section that follows, we define the discrete function spaces, we define the notion of a discrete inner product on these spaces and discuss possible methods of construction, and finally we define the discrete differential operators that act on these spaces.

In the third section we demonstrate that any choice of inner product yields operators that satisfy certain discrete vector calculus analogues and in particular a discrete Helmholtz orthogonality. This generalizes the results from [10] and uses techniques from [13]. Following recent work in finite element theory, see [1], we demonstrate how these properties can be placed in the framework of exact sequences.

In the fourth section we discuss the inner products on the edge spaces in greater depth. We analyze their accuracy as $L^{2}$ approximations and prove certain unique properties of the covolume inner product that make it particularly well suited for numerical work.

\section{Finite SPACES AND DisCRETE OPERATORS}

This section is devoted to the construction of discretizations of the differential operators divergence, curl, and gradient and of the finite-dimensional function spaces on which they act. We restrict our attention to $\mathbb{R}^{2}$ although the same formulations can be shown in three dimensions [18]. The discrete operators developed in this section generalize and include those found in Nicolaides [13] and Hyman and Shashkov [7,8].

We begin by defining certain necessary notation for a triangular mesh $\Omega_{h}$ in $\mathbb{R}^{2}$. On a simply connected polygonal domain $\Omega$ we place a triangular mesh $\Omega_{h}$. Denote by $\vec{z}_{k}$ for $k=1, \ldots, z$ the ordered nodes of the mesh. This ordering induces an orientation on the edges $\sigma_{i}$ for $i=1, \ldots, e$ and we write $h_{i}$ for the length of $\sigma_{i}$. Let $t_{j}$ for $j=1, \ldots, p$ denote the triangles of the mesh and $\left|t_{j}\right|$ their areas.

We assume the interior nodes, numbering $z_{0}$, are ordered first and likewise the interior edges $i=1, \ldots, e_{0}$ and note that the number of boundary edges and the number of boundary nodes is equal, i.e. $z-z_{0}=e-e_{0}$.

If the triangulation, $\Omega_{h}$, is a Delaunay triangulation then we introduce the following additional notation. With each node $\vec{z}_{k}$ we associate a region called a cotriangle or covolume region. This region is constructed by connecting the circumcenters of the triangles adjacent to the node. The resulting polygon is denoted $t_{k}^{\prime}$ and its area $\left|t_{k}^{\prime}\right|$. Each line segment connecting two adjacent circumcenters bisects an edge $\sigma_{i}$. This coedge will be written $\sigma_{i}^{\prime}$ and its length $h_{i}^{\prime}$. 
Finally, with each triangle $t_{j}$ we associate a point $\vec{p}_{j}$. There are a number of possible choices for the placement of this point inside the triangle, or possibly outside. These choices include the circumcenter and the centroid but may also be arbitrary.

\subsection{Finite-dimensional function spaces}

On the triangulation $\Omega_{h}$ there are four different finite-dimensional function spaces. Two of these discrete function spaces serve to approximate vector-valued functions. They are associated with the edges. The other two discrete function spaces are associated with either the nodes $\vec{z}_{k}$ or the triangles $t_{j}$.

The differential operator curl acts on three-dimensional vector fields. Therefore, when necessary, a planar vector field $\vec{u}(x, y)$ will be written $\vec{u}(x, y, z)=\left(u_{1}(x, y), u_{2}(x, y), 0\right)$ so that we can make sense of curl $\vec{u}$. Similarly, the resulting vector field curl $\vec{u}=\left(0,0, \frac{\partial u_{2}}{\partial x}-\frac{\partial u_{1}}{\partial y}\right)$ can be considered a scalar-valued function on the plane.

The first of the two discrete scalar-valued function spaces is the triangle space, denoted $\mathcal{T}$. It is a real vector space of dimension $p$. For an element $\hat{\phi}$ in $\mathcal{T}$ we associate the value $\hat{\phi}_{j}$ with the point $\vec{p}_{j}$ in $\Omega_{h}$. To project a continuous scalar-valued function $\phi$ on $\Omega$ onto the space $\mathcal{T}$ one can consider either a pointwise evaluation at $\vec{p}_{j}$, or an average of $\phi$ over $t_{j}$.

The other discrete scalar-valued function space is the nodal space $\mathcal{N}$ which is a real vector space of dimension $z$. For an element $\hat{\psi}$ in $\mathcal{N}$ we associate the value $\hat{\psi}_{k}$ with the node $\vec{z}_{k}$ in $\Omega_{h}$. The interior nodal space, the restriction of $\mathcal{N}$ to the interior nodes, will be denoted $\mathcal{N}_{0}$. It is a real vector space of dimension $z_{0}$. Projections of a continuous scalar-valued function $\psi$ on $\Omega$ onto $\mathcal{N}$ may similarly be performed by either sampling or averaging over the covolume region $t_{k}^{\prime}$. Either $\hat{\psi}_{k}=\psi\left(\vec{z}_{k}\right)$ or $\hat{\psi}_{k}=\frac{1}{\left|t_{k}^{\prime}\right|} \int_{t_{k}^{\prime}} \psi \mathrm{d} A$.

The two different discrete vector field spaces on $\Omega_{h}$ both associate values with the edges. We introduce a local coordinate frame and its subsequent notation for each edge $\sigma_{i}$. Let $\vec{x}_{i}$ be the midpoint of the edge and define $\vec{\tau}_{i}$ to be the unit vector tangent to the edge in the positive direction. The unit vector normal to the edge, $\vec{n}_{i}$, is aligned so that $\left(\vec{n}_{i}, \vec{\tau}_{i}\right)$ is a right-handed system.

The normal edge space $\mathcal{M}$ is a real vector space of dimension $e$. For an element $\hat{w}$ in $\mathcal{M}$ the value $\hat{w}_{i} \in \mathbb{R}$ is associated with the normal vector $\hat{w}_{i} \vec{n}_{i}$ at the point $\vec{x}_{i}$. The interior normal edge space $\mathcal{M}_{0}$ is the restriction of $\mathcal{M}$ to the interior edges. It is a vector space of dimension $e_{0}$. We consider two different projections of a continuous vector field $\vec{w}$ on $\Omega$ onto $\mathcal{M}$ (and similarly for $\mathcal{M}_{0}$ ). Either $\hat{w}_{i}$ is a pointwise evaluation or an edge average. So that $\hat{w}_{i}=\vec{w}\left(\vec{x}_{i}\right) \cdot \vec{n}_{i}$ or $\hat{w}_{i}=\frac{1}{h_{i}} \int_{\sigma_{i}} \vec{w} \cdot \vec{n}_{i} \mathrm{~d} l$.

The tangential edge space $\mathcal{E}$ is a real vector space of dimension $e$. For an element $\hat{u}$ in $\mathcal{E}$ the value $\hat{u}_{i} \in \mathbb{R}$ is associated with the tangent vector $\hat{u}_{i} \vec{\tau}_{i}$ at the point $\vec{x}_{i}$. The interior tangential edge space $\mathcal{E}_{0}$ is the restriction of $\mathcal{E}$ to the interior edges. It is a real vector space of dimension $e_{0}$. We again consider the following two projections of a continuous vector field $\vec{u}$ on $\Omega$ onto $\mathcal{E}$ (and similarly for $\mathcal{E}_{0}$ ): either $\hat{u}_{i}=\vec{u}\left(\vec{x}_{i}\right) \cdot \vec{\tau}_{i}$ or $\hat{u}_{i}=\frac{1}{h_{i}} \int_{\sigma_{i}} \vec{u} \cdot \vec{\tau}_{i} \mathrm{~d} l$.

\subsection{The primary operators}

We approximate the three differential operators divergence, curl and gradient. Given the discrete spaces defined above there is a very natural and common way to approximate these three operators through the use of their integral formulations.

For a continuous scalar-valued function $\psi$ we approximate $\operatorname{grad} \psi\left(\vec{x}_{i}\right) \cdot \vec{\tau}_{i}$. In integral form we have

$$
\operatorname{grad} \psi\left(\vec{x}_{i}\right) \cdot \vec{\tau}_{i} \approx \frac{1}{h_{i}} \int_{\sigma_{i}} \operatorname{grad} \psi \cdot \vec{\tau}_{i} \mathrm{~d} s \approx \frac{1}{h_{i}}\left(\psi\left(\vec{z}_{k+1}\right)-\psi\left(\vec{z}_{k}\right)\right) .
$$

This directional derivative acts naturally on the space of nodal scalar values in the oriented direction of an edge. Thus the discrete operator $G: \mathcal{N} \longrightarrow \mathcal{E}$ acts on $\hat{\psi} \in \mathcal{N}$ as follows:

$$
(G \hat{\psi})_{i}=\frac{\hat{\psi}_{k+1}-\hat{\psi}_{k}}{h_{i}}
$$



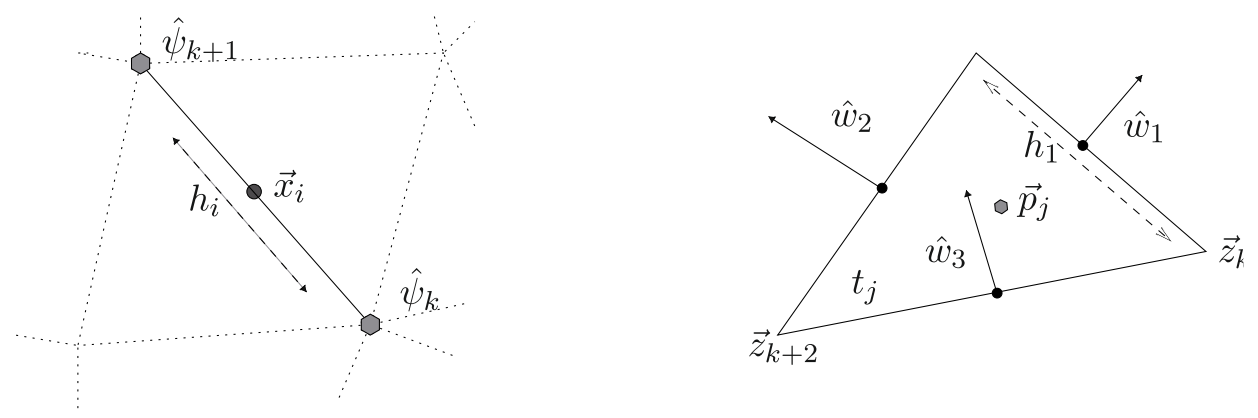

FiguRE 1. Discrete gradient and divergence operator stencils.

The divergence operator is approximated using the integral formulation of the operator found in the divergence theorem. For a triangle $t$, let $\vec{\nu}$ be the unit outward normal vector to the boundary. An approximation of the divergence via the divergence theorem is

$$
\operatorname{div} \vec{w}(\vec{p}) \approx \frac{1}{|t|} \int_{t} \operatorname{div} \vec{w} \mathrm{~d} A=\frac{1}{|t|} \int_{\partial t} \vec{w} \cdot \vec{\nu} \mathrm{d} l \approx \frac{1}{|t|} \sum_{i=1}^{3} \pm h_{i}\left(\vec{w}\left(\vec{x}_{i}\right) \cdot \vec{n}_{i}\right)
$$

This operator acts on the normal (to the edge) components of a vector field and approximates the divergence at a point $\vec{p}_{j}$ in the triangle. For the edge orientation depicted in Figure 1, the discrete operator $D: \mathcal{M} \longrightarrow \mathcal{T}$ acts on $\hat{w}$ by

$$
(D \hat{w})_{j}=\frac{1}{\left|t_{j}\right|}\left(h_{1} \hat{w}_{1}+h_{2} \hat{w}_{2}-h_{3} \hat{w}_{3}\right) .
$$

The curl operator is also approximated using an integral formulation, in this case Stokes' Theorem. Let $\vec{k}=(0,0,1)$ and $\vec{s}$ be the positively oriented unit tangent vector along the boundary of the triangle $t$. Then the approximation is

$$
\operatorname{curl} \vec{u}(\vec{p}) \cdot \vec{k} \approx \frac{1}{|t|} \int_{t} \operatorname{curl} \vec{u} \cdot \vec{k} \mathrm{~d} A=\frac{1}{|t|} \int_{\partial t} \vec{u} \cdot \vec{s} \mathrm{~d} l \approx \frac{1}{|t|} \sum_{i=1}^{3} \pm h_{i}\left(\vec{u}\left(\vec{x}_{i}\right) \cdot \vec{\tau}_{i}\right)
$$

Thi s operator acts on the tangential (to the edge) components of a vector field $\vec{u}$ and approximates the curl at the point $\vec{p}_{j}$. For the edge orientation depicted in Figure 2, the discrete operator $C: \mathcal{E} \longrightarrow \mathcal{T}$ acts on $\hat{u}$ by

$$
(C \hat{u})_{j}=\frac{1}{\left|t_{j}\right|}\left(h_{1} \hat{u}_{1}+h_{2} \hat{u}_{2}-h_{3} \hat{u}_{3}\right) .
$$

There is one more operator to consider as a primary operator and to do this we view the finite-dimensional scalar-valued function space $\mathcal{N}$ as a vector-valued function space with only a $\vec{k}$ component. Because a vector field $\vec{\omega}=(0,0, \omega)=\omega \vec{k}$ can be projected onto $\hat{\omega} \in \mathcal{N}$ we can, when necessary, consider elements of $\mathcal{N}$ to be discrete vector-valued functions. This interpretation leads to another discrete curl operator.

If we place a $h_{i} \times h_{i}$ square surface $S$ on the edge $\sigma_{i}$ so that the vector $\vec{n}_{i}$ normal to the edge is also normal to the surface then we can use Stokes' Theorem to approximate curl $\vec{\omega}\left(\vec{x}_{i}\right) \cdot \vec{n}_{i}$.

Since only two of the edges contribute to the circulation of $\vec{\omega}$ about the surface $S$ the approximation of $\operatorname{curl} \vec{\omega}\left(\vec{x}_{i}\right) \cdot \vec{n}_{i}$ reduces to $\frac{1}{h_{i}}\left(\hat{\omega}_{k+1}-\hat{\omega}_{k}\right)$. Therefore define $K: \mathcal{N} \longrightarrow \mathcal{M}$ to be

$$
(K \hat{\omega})_{i}=\frac{\hat{\omega}_{k+1}-\hat{\omega}_{k}}{h_{i}}
$$



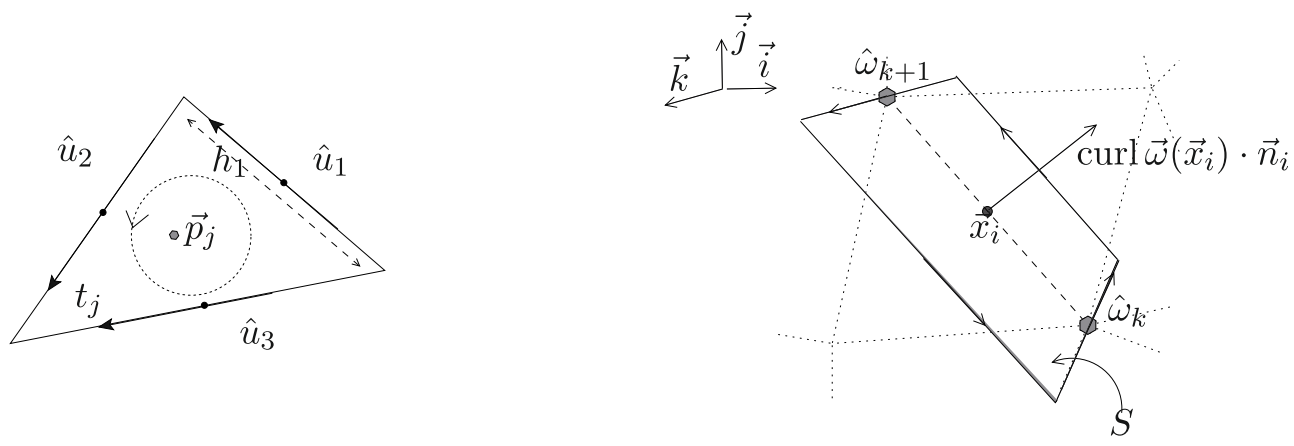

Figure 2. Discrete curl operators' stencils.

The discretization of each of these four primary discrete operators relies on local values from the discrete spaces $\mathcal{E}, \mathcal{M}, \mathcal{N}$, and $\mathcal{T}$. For this reason, we borrow the nomenclature from finite difference methods and refer to these operators as having local stencils.

The discrete operators thus defined have domains and ranges $G: \mathcal{N} \longrightarrow \mathcal{E}, K: \mathcal{N} \longrightarrow \mathcal{M}, C: \mathcal{E} \longrightarrow \mathcal{T}$, and $D: \mathcal{M} \longrightarrow \mathcal{T}$ and therefore the only compositions that we can perform are $C G$ and $D K$.

With respect to the standard basis in $\mathbb{R}^{n}$ (for appropriate $n$ ) we can represent these operators as matrices. So that, for example, the operator $G$ is written as an $e \times z$ matrix with two non-zero entries $+\frac{1}{h_{i}}$ and $-\frac{1}{h_{i}}$ in each row $i$. Due to the one-to-one orientation preserving correspondence between the spaces $\mathcal{E}$ and $\mathcal{M}$ the matrices that represent the operators $G$ and $K$ are numerically identical as are the matrices for $C$ and $D$.

These primary operators are the same for every method in the class of algorithms that we investigate. The operators that we define in Section 2.4 (the dual operators) which complete the collection of discrete operators needed (to solve div-curl systems, Maxwell's equations, and diffusion) will differ from each other due to their dependence on a choice of discrete inner product (chosen to approximate the integral inner products of $L^{2}(\Omega)$ and $\left.\left(L^{2}(\Omega)\right)^{2}\right)$ on the discrete function spaces $\mathcal{M}, \mathcal{E}, \mathcal{N}$, and $\mathcal{T}$.

\subsection{The inner products}

We approximate the $L^{2}(\Omega)$ and $\left(L^{2}(\Omega)\right)^{2}$ inner products by suitably extending an element in $\mathcal{E}, \mathcal{T}, \mathcal{N}$, and $\mathcal{M}$ to a full vector or scalar-valued field on $\Omega$. The continuous inner product on these extensions can be represented as a finite dimensional bilinear form and thus there is a matrix representation. The bases with respect to which we represent these matrices are again the standard bases in $\mathbb{R}^{n}$ for the appropriate $n$. In the spaces $\mathcal{E}, \mathcal{T}, \mathcal{N}$ and $\mathcal{M}$ these basis vectors correspond to one edge, one triangle, or one node being nonzero. Each choice of extension of an element of a discrete space yields a different discrete inner product on the space.

For two vectors $\vec{a}$ and $\vec{b}$ in $\mathbb{R}^{n}$ we will denote by $(\vec{a}, \vec{b})$ and $\vec{a} \cdot \vec{b}$ the standard Euclidean inner product $\sum_{i=1}^{n} a_{i} b_{i}$. All other inner products, e.g. $S$, will be written $[\cdot, \cdot]_{S}$.

Given two elements $\hat{\rho}$ and $\hat{\phi}$ in $\mathcal{T}$ we construct piecewise constant scalar-valued functions $\rho_{h}$ and $\phi_{h}$ by extending the values $\hat{\rho}_{j}$ and $\hat{\phi}_{j}$ throughout the triangle $t_{j}$. With the triangle areas, $\left|t_{j}\right|$, we let $T$ be the $p \times p$ diagonal matrix with $T_{j j}=\left|t_{j}\right|$. The representation of the approximation to the continuous inner product is then

$$
[\hat{\rho}, \hat{\phi}]_{\mathcal{T}}:=\hat{\rho} \cdot T \hat{\phi}=\sum_{j=1}^{p}\left|t_{j}\right| \hat{\rho}_{j} \hat{\phi}_{j}=\int_{\Omega} \rho_{h} \phi_{h} \mathrm{~d} A .
$$

Given two elements $\hat{\omega}$ and $\hat{\psi}$ in $\mathcal{N}$ we again construct piecewise constant scalar-valued functions $\omega_{h}$ and $\psi_{h}$ in $\Omega_{h}$. Since the value $\hat{\omega}_{k}$ is associated with the node $\vec{z}_{k}$ the region to which it should be extended is not as apparent as in the case of the triangle extension for $\mathcal{T}$. The only requirement that we make is that the regions $N_{k}$ associated with the nodes (and thus the extension) should partition $\Omega_{h}$, i.e. they should not overlap and their 


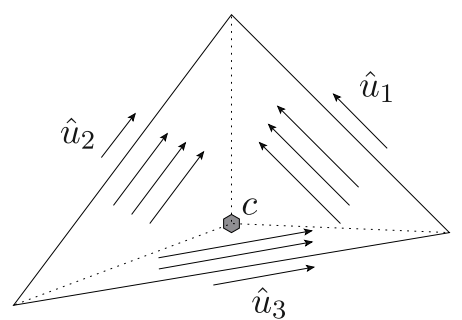

Covolume extension $\vec{u}_{h}$

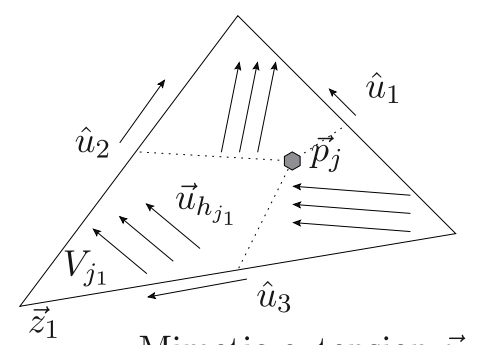

Mimetic extension $\vec{u}_{h}$

Figure 3. Vector field extensions.

union should cover the entire domain. In the case of a Delaunay triangulation an obvious choice for $N_{k}$ would be the covolume region. This is the domain about the node that results from connecting the circumcenter of each adjacent triangle.

Let $N$ be the $z \times z$ diagonal matrix with $N_{k k}=\left|N_{k}\right|$, where $\left|N_{k}\right|$ is the area of the region $N_{k}$. These areas or nodal weights yield a representation of the approximation to the continuous inner product:

$$
[\hat{\omega}, \hat{\psi}]_{\mathcal{N}}:=\hat{\omega} \cdot N \hat{\psi}=\sum_{k}\left|N_{k}\right| \hat{\omega}_{k} \hat{\psi}_{k}=\int_{\Omega} \omega_{h} \psi_{h} \mathrm{~d} A
$$

The $z_{0} \times z_{0}$ matrix that represents the restriction of the inner product $N$ to the space $\mathcal{N}_{0}$ will be $\operatorname{denoted} N_{0}$.

The inner product on the tangential edge space, $\mathcal{E}$, requires a vector-field reconstruction and there are many suitable methods of extending an element $\hat{u}$ in $\mathcal{E}$ to a full vector-valued field $\vec{u}_{h}$ in $\Omega$. Each method that we consider shares the following property: the value of $\vec{u}_{h}$ at a point in a triangle $t_{j}$ depends only on the three edge values $\left\{\hat{u}_{j_{1}}, \hat{u}_{j_{2}}, \hat{u}_{j_{3}}\right\}$ of the triangle.

- Covolume inner product:

This method of extending $\hat{u}$ into the triangle $t$ uses the circumcenter of the triangle $c$ and three triangle subregions created by connecting the circumcenter to the nodes. Each subregion is bordered by only one edge and thus one value $\hat{u}_{i}$. The vector $\sqrt{2} \hat{u}_{i} \vec{\tau}_{i}$ is extended throughout the region. This results in a vector field $\vec{u}_{h}$ that is constant in each subregion. See Figure 3.

This discrete inner product can be represented, with respect to the standard basis on $\mathcal{E}$, as an $e \times e$ diagonal matrix $W$ with $W_{i i}=h_{i} h_{i}^{\prime}$. Recall that $h_{i}^{\prime}$ is the length of the coedge $\sigma_{i}^{\prime}$.

We write

$$
[\hat{u}, \hat{v}]_{W}:=\hat{u} \cdot W \hat{v}=\sum_{i} \hat{u}_{i} \hat{v}_{i} h_{i} h_{i}^{\prime}=\int_{\Omega} \vec{u}_{h} \cdot \vec{v}_{h} \mathrm{~d} A .
$$

- Mimetic inner product:

This method of extending $\hat{u}$ relies on choosing three nodal subregions of each triangle $t_{j}$ with areas (or nodal weights) $\left\{V_{j_{1}}, V_{j_{2}}, V_{j_{3}}\right\}$. Each subregion is to be bordered by two edges. Throughout the subregion with area $V_{j_{1}}$ and adjacent edges $\sigma_{i}$ and $\sigma_{i+1}$ the constant vector field $\vec{u}_{h_{j_{1}}}$ is extended. This is the unique vector that satisfies $\vec{u}_{h_{j_{1}}} \cdot \vec{\tau}_{i}=\hat{u}_{i}$ and $\vec{u}_{h_{j_{1}}} \cdot \vec{\tau}_{i+1}=\hat{u}_{i+1}$. Piecing together these constant vector fields yields a full vector field $\vec{u}_{h}$ whose projections onto the tangential vectors of the edges agrees with $\hat{u}$. See Figure 3.

With respect to the standard basis on $\mathcal{E}$ we can represent this inner product as a matrix $\widetilde{W}$ and write

$$
[\hat{u}, \hat{v}]_{\widetilde{W}}=\hat{u} \cdot \widetilde{W} \hat{v}=\sum_{j=1}^{p} \sum_{k=1}^{3}\left(\vec{u}_{h_{j_{k}}} \cdot \vec{v}_{h_{j_{k}}}\right) V_{j_{k}}=\int_{\Omega} \vec{u}_{h} \cdot \vec{v}_{h} \mathrm{~d} A .
$$


- Raviart-Thomas/Nedelec method:

This method of extension is unique among the methods mentioned here in that the resulting $\vec{u}_{h}$ is not a piecewise constant vector field over subregions of each triangle. Here a linear vector field is extended throughout the triangle. With only three pieces of information about the vector field on a given triangle a full linear vector field is not uniquely determined. Thus the extension is restricted to a particular three-dimensional subspace of linear vector fields. For $a_{1}, a_{2}, b \in \mathbb{R}$ this subspace of linear vector fields is

$$
\mathcal{R}_{t}:=\left\{L: \mathbb{R}^{2} \longrightarrow \mathbb{R}^{2}: L(x, y)=\left(a_{1}-b y, a_{2}+b x\right)\right\}
$$

Given $\left\{\hat{u}_{1}, \hat{u}_{2}, \hat{u}_{3}\right\}$ in a triangle $t$ one can uniquely solve for a vector field in $\mathcal{R}_{t}$ that satisfies $L\left(\vec{x}_{i}\right) \cdot \vec{\tau}_{i}=\hat{u}_{i}$ for $i=1,2,3$. In each triangle the resulting vector field is divergence-free and the tangential component of $L(\vec{x})$ along an edge is constant. As this extension is performed on neighboring triangles the tangential components of the global reconstructed vector field will be continuous across the edges while the normal components will not be $[12,17]$.

For each matrix $X$ representing a discrete inner product on $\mathcal{E}$ we denote by $X_{0}$ the $e_{0} \times e_{0}$ matrix that results from deleting the rows and columns in $X$ that correspond to the boundary edges. This matrix $X_{0}$ represents the discrete inner product on $\mathcal{E}_{0}$.

The finite vector spaces $\mathcal{M}$ and $\mathcal{E}$ share the same possibilities for discrete inner products. The methods of extending the values of an element $\hat{w} \in \mathcal{M}$ throughout the triangle $t$ are in all but one case the same as the methods of extending $\hat{u} \in \mathcal{E}$.

The covolume inner product on $\mathcal{M}$ is exactly the same as the covolume inner product on $\mathcal{E}$. Similarly, the mimetic inner product is the same on both of these spaces. However, the Raviart-Thomas/Nedelec method of extending the three edge values $\left(\hat{w}_{1}, \hat{w}_{2}, \hat{w}_{3}\right)$ throughout a triangle $t$ differs. For $\mathcal{M}$ the three-dimensional subspace of linear vector fields is $\mathcal{P}_{t}:=\left\{L: \mathbb{R}^{2} \longrightarrow \mathbb{R}^{2}: L(x, y)=\left(a_{1}+b x, a_{2}-b y\right)\right\}$.

Given $\left\{\hat{w}_{1}, \hat{w}_{2}, \hat{w}_{3}\right\}$ in a triangle $t$ one can uniquely solve for a vector field in $\mathcal{P}_{t}$ that satisfies $L\left(\vec{x}_{i}\right) \cdot \vec{n}_{i}=\hat{w}_{i}$ for $i=1,2,3$. The resulting field is curl-free in the triangle $t$ and the normal component of $L(\vec{x})$ along an edge is constant. As this extension is performed on neighboring triangles the normal components across the edges will be continuous while the tangential components will not $[12,17]$.

We denote by $M$ a generic matrix representation of an inner product on $\mathcal{M}$ and the restriction of the discrete inner product to the subspace $\mathcal{M}_{0}$ will be denoted $M_{0}$. This $e_{0} \times e_{0}$ matrix results from deleting the rows and columns in $M$ corresponding to boundary edges.

\subsection{The dual operators}

Our goal now is to complete our collection of discrete operators. The primary operators alone restrict our ability to make all desired compositions, they do not act on all appropriate discrete spaces and they can not satisfy the discrete integral identity analogs which we require. The dual operators that we derive in this section combined with the primary operators achieve these three goals.

Following the mimetic method, the construction of the dual operators relies on a discrete version of the integration by parts identities that exist for continuous vector and scalar-valued functions and the $L^{2}$ inner products. Let $\vec{u}$ and $\vec{v}$ be vector fields on $\Omega$ and $\phi$ a scalar-valued function. Let $\vec{\nu}$ be the outward unit vector normal to the boundary of $\Omega$ and $\vec{\tau}$ the unit vector tangent to the boundary aligned so that $(\vec{\nu}, \vec{\tau})$ forms a right hand system. Integration by parts gives the following two identities:

$$
\begin{aligned}
& \int_{\Omega} \phi \operatorname{div} \vec{u} \mathrm{~d} V=-\int_{\Omega} \vec{u} \cdot \operatorname{grad} \phi \mathrm{d} V+\int_{\partial \Omega} \phi(\vec{u} \cdot \vec{\nu}) \mathrm{d} A . \\
& \int_{\Omega} \vec{v} \cdot \operatorname{curl} \vec{u} \mathrm{~d} V=\int_{\Omega} \vec{u} \cdot \operatorname{curl} \vec{v} \mathrm{~d} V-\int_{\partial \Omega} \vec{v} \cdot(\vec{u} \times \vec{\nu}) \mathrm{d} A .
\end{aligned}
$$


The primary discrete operators $G, C, D$, and $K$ are independent of the choice of discrete inner products on the spaces $\mathcal{E}, \mathcal{M}, \mathcal{N}$, and $\mathcal{T}$. The dual operators will differ depending on the choice of inner product. The following construction is shown for arbitrary inner products $X, M, N$, and $T$ with restricted inner products $X_{0}$ on $\mathcal{E}_{0}, M_{0}$ on $\mathcal{M}_{0}$ and $N_{0}$ on $\mathcal{N}_{0}$.

\subsubsection{The divergence and gradient as adjoint operators}

The primary operator $G: \mathcal{N} \longrightarrow \mathcal{E}$ can be restricted to $G_{0}: \mathcal{N}_{0} \longrightarrow \mathcal{E}_{0}$. The resulting $e_{0} \times z_{0}$ matrix representing $G_{0}$ is obtained from $G$ by deleting those columns and rows corresponding to boundary edges or boundary nodes.

If $\phi=0$ on the boundary of $\Omega$ then the boundary integral in the right side of equation (2.1) will vanish. The function $\phi$ can be projected onto the subspace $\mathcal{N}_{0}$ and we can approximate the right hand side of equation (2.1) for $\vec{u}$ projected onto $\mathcal{E}_{0}$ as

$$
-\int_{\Omega} \vec{u} \cdot \operatorname{grad} \phi \mathrm{d} V+\int_{\partial \Omega} \phi(\vec{u} \cdot \vec{\nu}) \mathrm{d} A=-\int_{\Omega} \vec{u} \cdot \operatorname{grad} \phi \mathrm{d} V \approx-\left[\hat{u}, G_{0} \hat{\phi}\right]_{X_{0}} .
$$

We can not currently make sense of the left hand side of equation (2.1) for $\hat{u} \in \mathcal{E}_{0}$ since $D$ acts on $\mathcal{M}$. A discrete approximation to the divergence which did act on $\mathcal{E}_{0}$, say $D^{*}: \mathcal{E}_{0} \longrightarrow \mathcal{N}_{0}$, would allow us to discretize the left side of equation (2.1) as

$$
\int_{\Omega} \phi \operatorname{div} \vec{u} \mathrm{~d} V \approx\left[\hat{\phi}, D^{*} \hat{u}\right]_{\mathcal{N}_{0}} .
$$

To ensure a discrete analog of this vector calculus identity we define the dual operator $D^{*}: \mathcal{E}_{0} \longrightarrow \mathcal{N}_{0}$ to be the unique operator which satisfies for all $\hat{\phi} \in \mathcal{N}_{0}$ and all $\hat{u} \in \mathcal{E}_{0}$ the discrete version of the integral identity (2.1). That is

$$
\hat{\phi} \cdot N_{0} D^{*} \hat{u}=\left[\hat{\phi}, D^{*} \hat{u}\right]_{\mathcal{N}_{0}}=-\left[\hat{u}, G_{0} \hat{\phi}\right]_{X_{0}}=\left(G_{0} \hat{\phi}\right) \cdot X_{0} \hat{u} .
$$

Therefore $D^{*}:=-N_{0}^{-1} G_{0}^{T} X_{0}$.

If instead we consider $\hat{\phi} \in \mathcal{T}$ and $\hat{u} \in \mathcal{M}_{0}$ then the primary discrete divergence operator allows us to approximate the integral in the left hand side of equation (2.1) as

$$
\int_{\Omega} \phi \operatorname{div} \vec{u} \mathrm{~d} V \approx\left[D_{0} \hat{u}, \hat{\phi}\right]_{\mathcal{T}}
$$

Here $D_{0}$ is the restriction of $D$ to $M_{0}$ whose $p \times e_{0}$ matrix representation is obtained from $D$ by deleting those columns corresponding to boundary edges. Thus $D_{0}: \mathcal{M}_{0} \longrightarrow \mathcal{T}$.

This leads to the dual discrete gradient $G^{*}: \mathcal{T} \longrightarrow \mathcal{M}_{0}$. This $G^{*}$ is the unique operator satisfying

$$
D_{0} \hat{u} \cdot T \hat{\phi}=\left[D_{0} \hat{u}, \hat{\phi}\right]_{\mathcal{T}}=-\left[\hat{u}, G^{*} \hat{\phi}\right]_{\mathcal{M}_{0}}=-\hat{u} \cdot M_{0}\left(G^{*} \hat{\phi}\right) .
$$

Thus the dual operator $G^{*}:=-M_{0}^{-1} D_{0}^{T} T$.

\subsubsection{The curl as a self-adjoint operator}

As above, we consider the restriction of $C: \mathcal{E} \longrightarrow \mathcal{T}$ to the domain $\mathcal{E}_{0}$ by deleting the columns in the matrix corresponding to boundary edges. We denote this resulting $p \times e_{0}$ matrix operator $C_{0}$.

A vector field $\vec{u}$ satisfying $\vec{u} \cdot \vec{\tau}=0$ on the boundary of $\Omega$ can be projected onto $\mathcal{E}_{0}$. This condition is equivalent to $\vec{u} \times \vec{\nu}=0$ on the boundary of $\Omega$ which results in the boundary integral in equation (2.2) being zero. 
If $\vec{v}$ has only a $\vec{k}$ component, $\vec{v}=(0,0, v)$, then we can project it onto the finite space $\mathcal{T}$ and the left hand side of equation $(2.2)$ can be approximated using the discrete inner product on $\mathcal{T}$. Thus we have

$$
\int_{\Omega} \vec{v} \cdot \operatorname{curl} \vec{u} \mathrm{~d} V \approx\left[\hat{v}, C_{0} \hat{u}\right]_{\mathcal{T}}
$$

Since $\hat{u}$ is an element of $\mathcal{E}_{0}$ the right hand side of equation (2.2) must be discretized using the discrete inner product $X_{0}$ on $\mathcal{E}_{0}$. We let $C^{*}$ be the formal adjoint operator that acts on $\mathcal{T}$ and has range $\mathcal{E}_{0}$ :

$$
\int_{\Omega} \vec{v} \cdot \operatorname{curl} \vec{u} \mathrm{~d} V \approx\left[\hat{v}, C_{0} \hat{u}\right]_{\mathcal{T}}=\left[\hat{u}, C^{*} \hat{v}\right]_{X_{0}} \approx \int_{\Omega} \vec{u} \cdot \operatorname{curl} \vec{v} \mathrm{~d} V .
$$

We require that for all $\hat{v} \in \mathcal{T}$ and all $\hat{u} \in \mathcal{E}_{0}$ the operator $C^{*}$ satisfies

$$
C_{0} \hat{u} \cdot T \hat{v}=\left[C_{0} \hat{u}, \hat{v}\right]_{\mathcal{T}}=\left[\hat{u}, C^{*} \hat{v}\right]_{X_{0}}=\hat{u} \cdot X_{0} C^{*} \hat{v}
$$

Therefore this dual curl operator $C^{*}:=X_{0}^{-1} C_{0}^{T} T$.

To define the dual operator to $K$ we again restrict $K$ to $\mathcal{N}_{0}$ with range $\mathcal{M}_{0}$ by deleting those rows and columns in $K$ corresponding to boundary edges and boundary nodes. Here we consider $\hat{u}$ in $\mathcal{M}_{0}$.

If the vector field $\vec{v}=(0,0, v)$ vanishes on $\partial \Omega$ then the boundary integral in equation (2.2) is zero. Such a $\vec{v}$ can be projected onto the discrete space $\mathcal{N}_{0}$ and the discrete approximation of the integral identity is

$$
\int_{\Omega} \vec{v} \cdot \operatorname{curl} \vec{u} \mathrm{~d} V \approx\left[\hat{v}, K^{*} \hat{u}\right]_{\mathcal{N}_{0}}=\left[\hat{u}, K_{0} \hat{v}\right]_{\mathcal{M}_{0}} \approx \int_{\Omega} \vec{u} \cdot \operatorname{curl} \vec{v} \mathrm{~d} V .
$$

With the discrete inner products $N_{0}$ on $\mathcal{N}_{0}$ and $M_{0}$ on $\mathcal{M}_{0}$ this results in the other dual curl operator $K^{*}:=$ $N_{0}^{-1} K_{0}^{T} M_{0}$.

These four dual operators together with the four primary operators ensure every possible composition of differential operators has an analog at the discrete level. Moreover every finite function space can be acted on by an appropriate discrete operator. Additionally, these discrete operators and discrete spaces exhibit many important vector calculus analogs. These properties, essential to the analysis of the discrete differential systems of equations to which they are applied, are explored in the next section.

\section{Discrete vector Calculus properties}

The specific examples of vector calculus properties that transfer to these discrete operators and finite spaces include the compositions of particular differential operators that result in zero, integration by parts, and the existence of scalar and vector potentials. Moreover, with this structure in place other discrete analogs of continuous properties result. Conservation properties and decomposition theorems are particularly important in the study of partial differential equations and preserving them in the discrete system ensures everything from existence and uniqueness of solutions to stability.

This section describes the transfer of vector calculus identities of continuous functions and differential operators to discrete analogs on these finite spaces and discrete operators. The introduction of an exact sequence which encodes these discrete vector calculus analogs creates a useful framework with which to view these properties.

The preservation of these vector calculus identities on the discrete operators is independent of the choice of discrete inner products on the spaces $\mathcal{E}, \mathcal{N}, \mathcal{M}$, and $\mathcal{T}$ and we assume nothing about the inner product except that it is a symmetric positive definite bilinear form on the vector space $\mathbb{R}^{n}$ for the appropriate dimension $n$. 


\subsection{Composition of operators and existence of potentials}

For a continuous vector field $\vec{u}$ defined on a simply connected domain $\Omega$ the following composition of differential operators always results in zero: div curl $\vec{u}=0$. The discrete analog of this composition property requires both primary and dual operator compositions of the discrete divergence and curl operators to be zero. Thus we need to verify that for all $\hat{\omega} \in \mathcal{N}$ and $\hat{\rho} \in \mathcal{T}$ we have $D K \hat{\omega}=0$ and $D^{*} C^{*} \hat{\rho}=0$.

Similarly, the following composition acting on a continuous scalar-valued function $\phi$ is always zero: curl $\operatorname{grad} \phi=\overrightarrow{0}$. Here, the discrete analog requires that for all $\hat{\psi} \in \mathcal{N}$ and $\hat{\phi} \in \mathcal{T}$ we have $C G \hat{\psi}=0$ and $K^{*} G^{*} \hat{\phi}=0$.

The following theorem confirming this composition property of the primary curl and gradient operators is found in Nicolaides' paper [13].

Theorem 3.1. For all $\hat{\psi} \in \mathcal{N}$ and $\hat{\psi}_{0} \in \mathcal{N}_{0}, C G \hat{\psi}=0$ and $C_{0} G_{0} \hat{\psi}_{0}=0$.

By the construction of the dual operators the following corollary immediately follows.

Corollary 3.1. For all $\hat{\rho} \in \mathcal{T}, D^{*} C^{*} \hat{\rho}=0$.

For all $\hat{\omega} \in \mathcal{N}, D K \hat{\omega}=0$.

For all $\hat{\omega}_{0} \in \mathcal{N}_{0}, D_{0} K_{0} \hat{\omega}_{0}=0$.

For all $\hat{\phi} \in \mathcal{T}, K^{*} G^{*} \hat{\phi}=0$.

Together this theorem and its corollaries show that every possible discrete composition that approximates (div curl) and (curl grad) annihilates the corresponding vector or scalar-valued function approximation in the discrete spaces $\mathcal{N}$ and $\mathcal{T}$ upon which it acts.

A second important property of these compositions is the existence of scalar or vector-valued potential functions. For a continuous vector field $\vec{u}$ defined on the simply connected planar domain $\Omega$ we have that if curl $\vec{u}=0$ then $\vec{u}=\operatorname{grad} \phi$ for some scalar field $\phi$. Similarly, for a continuous vector field $\vec{v}$, if $\operatorname{div} \vec{v}=0$ then $\vec{v}=\operatorname{curl} \vec{w}$ for some vector field $\vec{w}$.

These functions $\phi$ and $\vec{w}$ are called potentials. The transfer of these properties to the discrete operators follows from this theorem also proved in [13].

Theorem 3.2. If $C \hat{u}=0$ then there exists $\hat{\psi} \in \mathcal{N}$ such that $G \hat{\psi}=\hat{u}$.

If $C_{0} \hat{u}=0$ then there exists $\hat{\psi} \in \mathcal{N}_{0}$ such that $G_{0} \hat{\psi}=\hat{u}$.

The corollary statements can be proved in the same manner as the above theorem: using a subspace dimensionality argument and Euler's formula for a triangulation with no holes.

Corollary 3.2. If $D^{*} \hat{u}=0$ then there exists $\hat{\phi} \in \mathcal{T}$ such that $C^{*} \hat{\phi}=\hat{u}$.

If $D \hat{v}=0$ then there exists $\hat{\omega} \in \mathcal{N}$ such that $K \hat{\omega}=\hat{v}$.

If $D_{0} \hat{v}=0$ then there exists $\hat{\omega} \in \mathcal{N}_{0}$ such that $K_{0} \hat{\omega}=\hat{v}$.

If $K^{*} \hat{v}=0$ then there exists $\hat{\rho} \in \mathcal{T}$ such that $G^{*} \hat{\rho}=\hat{v}$.

\subsection{The exact sequence}

The discrete analogs of vector calculus properties described above can be encoded in the larger framework of an exact sequence. This method of describing certain important properties of the finite-dimensional function spaces is often used in finite element methods for stability analysis [1].

In the case of Raviart-Thomas/Nedelec elements, the exactness of the sequence is inherited through the commutative diagram from the exactness properties of the continuous differential operators (viewed as operators on differential forms).

In our case, the exactness is built in from the definitions of the discrete operators, the choice of discrete spaces and the construction of the dual operators. In addition to the exactness properties the sequences capture the discrete analog of integration by parts.

These discrete features are expressed in Figure 4 (a similar diagram exists for the operators $K, D, G^{*}$ and $K^{*}$ acting on spaces $\mathcal{N}, \mathcal{M}$, and $\mathcal{T}$ ). Here the discrete function spaces are placed horizontally with the appropriate 


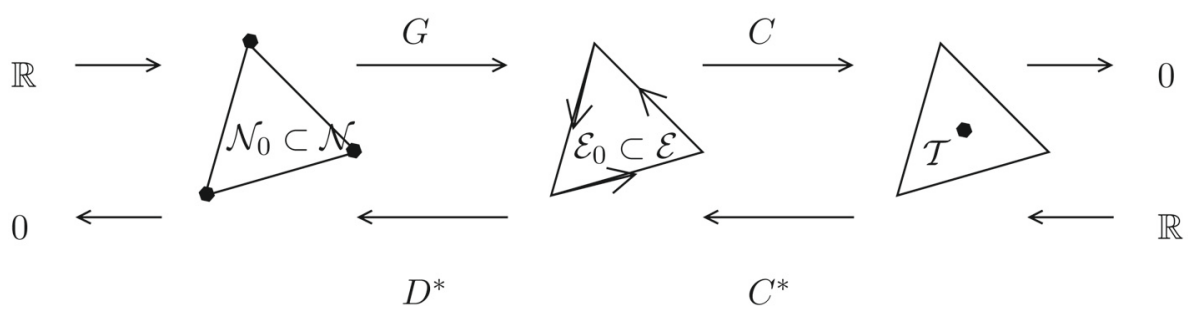

FiguRE 4. Exact sequence for discrete gradient and curl operators.

primary operators acting from left to right and arrows denoting their domains and ranges. The dual operators and their arrows from domains to ranges, act from right to left. In this way, the exactness of the sequence captures the null compositions and the existence of potentials. The dual operators, derived through the discrete inner products on the spaces, ensure the preservation of a discrete analog of integration by parts.

\subsection{The Helmholtz decomposition}

One very important consequence of the preservation of the above properties is the existence of the Helmholtz orthogonality property. On a simply connected domain $\Omega$ in $\mathbb{R}^{2}$ we consider two continuous vector fields $\vec{u}$ and $\vec{v}$. Let $\operatorname{div} \vec{u}=0$ and $\operatorname{curl} \vec{v}=0$. If either $(\vec{v} \cdot \vec{\tau})=0$ or $(\vec{u} \cdot \vec{\nu})=0$ on $\partial \Omega$ then the Helmholtz orthogonality property states that $[\vec{u}, \vec{v}]_{L^{2}(\Omega)}=0$.

Since $\operatorname{div} \vec{u}=0$ there exists a vector potential, $\vec{\omega}$, such that $\vec{u}=\operatorname{curl} \vec{\omega}$. Similarly, since curl $\vec{v}=0$, there is a scalar potential $\psi$ such that $\vec{v}=\operatorname{grad} \psi$. The importance of these potentials is shown in the following two calculations which demonstrate the orthogonality:

$$
\begin{aligned}
\int_{\Omega} \vec{u} \cdot \vec{v} \mathrm{~d} A & =\int_{\Omega} \operatorname{curl} \vec{\omega} \cdot \operatorname{grad} \psi \mathrm{d} A=-\int_{\Omega} \psi \operatorname{div} \operatorname{curl} \vec{\omega} \mathrm{d} A-\int_{\partial \Omega} \psi(\operatorname{curl} \vec{\omega} \cdot \vec{\nu}) \mathrm{d} A \\
& =\int_{\partial \Omega} \psi(\vec{u} \cdot \vec{\nu}) \mathrm{d} A .
\end{aligned}
$$

Equivalently, we can write

$$
\begin{aligned}
\int_{\Omega} \vec{u} \cdot \vec{v} \mathrm{~d} A & =\int_{\Omega} \operatorname{curl} \vec{\omega} \cdot \operatorname{grad} \psi \mathrm{d} A=\int_{\Omega} \vec{\omega} \cdot \operatorname{curl} \operatorname{grad} \psi \mathrm{d} A-\int_{\partial \Omega} \operatorname{grad} \psi \cdot(\vec{\omega} \times \vec{\nu}) \mathrm{d} A \\
& =\int_{\partial \Omega} \vec{v} \cdot(\vec{u} \times \vec{\nu}) \mathrm{d} A=-\int_{\partial \Omega} \vec{u} \cdot(\vec{v} \times \vec{\nu}) \mathrm{d} A .
\end{aligned}
$$

By the boundary condition hypothesis, one of these two expressions is zero. This orthogonality property relies on the vector calculus properties of integration by parts, composition theorems, and the existence of potentials. As these properties all have discrete analogs it follows that a discrete analog of the Helmholtz decomposition exists as well.

Lemma 3.1. (1) For $\hat{u}, \hat{v} \in \mathcal{E}_{0}$. If $C_{0} \hat{u}=0$ and $D^{*} \hat{v}=0$ then $[\hat{u}, \hat{v}]_{\mathcal{E}_{0}}=0$.

(2) For $\hat{u}, \hat{v} \in \mathcal{M}_{0}$. If $K^{*} \hat{u}=0$ and $D_{0} \hat{v}=0$ then $[\hat{u}, \hat{v}]_{\mathcal{M}_{0}}=0$.

Proof. This follows from Theorems 3.2.1, 3.2.2 and their corollaries.

The spaces $\mathcal{E}_{0}$ and $\mathcal{M}_{0}$ can thus be decomposed into a discrete divergence-free space and a discrete curl-free space. 
The discrete Helmholtz decomposition property is an essential part of the theory of this class of discrete approximations to partial differential equations. It not only captures important physical properties present in the continuous equations, it is used to determine the existence and uniqueness of solutions as well as analyze the convergence of the discrete solutions to the systems of equations to which these methods are applied [10,13,18].

The existence of this discrete decomposition is a direct consequence of the discrete vector calculus properties captured in the exact sequence diagram. The spaces, operators, and constructions described here are just one large collection that can be placed into the exact sequence framework. But any collection of spaces and operators that satisfy the exactness property and duality of operators that is encoded in the exact sequence will have a discrete Helmholtz decomposition.

\section{THE INNER PRODUCTS ON $\mathcal{E}$}

This section focuses on specific properties of and relationships among the discrete inner products on the tangential edge space $\mathcal{E}$. The matrices representing these inner products are analyzed and the covolume inner product is shown to have certain unique properties.

We restrict our attention to one triangle $t$ and the corresponding restriction of the space $\mathcal{E}$ to the threedimensional subspace we denote by $\mathcal{E}_{t}$. The vectors $\{(1,0,0),(0,1,0),(0,0,1)\}$, the standard basis in $\mathbb{R}^{3}$, on this subspace will be written $\left\{\hat{u}^{1}, \hat{u}^{2}, \hat{u}^{3}\right\}$.

The discrete inner products that act on this subspace with respect to the standard basis can be represented as $3 \times 3$ matrices. They will be described in more detail in conjunction with descriptions of the different methods of constructing $\vec{u}_{h}$. It is understood that they are always written with respect to the basis $\left\{\hat{u}^{1}, \hat{u}^{2}, \hat{u}^{3}\right\}$.

We introduce another basis for the space $\mathcal{E}_{t}$ to more easily analyze how well these discrete inner products approximate the $\left(L^{2}(t)\right)^{2}$ inner product and in particular whether they yield exact approximations when acting on projections (onto $\mathcal{E}_{t}$ ) of constant vector fields in $\mathbb{R}^{2}$.

For computational purposes we consider the triangle $t$ to be aligned at the origin with the edge $\sigma_{1}$ along the $x$-axis. The constant vector fields $\vec{e}:=(1,0)$ and $\vec{f}:=(0,1)$ serve as a basis for all constant vector fields in $\mathbb{R}^{2}$ defined on the triangle. Their projections onto $\mathcal{E}_{t}$ will be denoted $\hat{e}$ and $\hat{f}$. See Figure 5 .

The third vector that we choose to complete this alternate basis for $\mathcal{E}_{t}$ is $\hat{h}:=\left(h_{1}, h_{2}, h_{3}\right)$. It is important to note that in the standard Euclidean inner product on $\mathbb{R}^{3}$ the vectors $\hat{e}$ and $\hat{f}$ are not orthogonal but that $\hat{h}$ is orthogonal to both.

A few trigonometric identities show this:

$$
\begin{gathered}
(\hat{h}, \hat{e})=h_{1}-h_{2} \cos \phi_{3}-h_{3} \cos \phi_{2}=0 \text { and } \\
(\hat{h}, \hat{f})=h_{2} \sin \phi_{3}-h_{3} \sin \phi_{2}=0 .
\end{gathered}
$$

The vector fields $\vec{e}$ and $\vec{f}$ are orthogonal with respect to the $\left(L^{2}(t)\right)^{2}$ inner product and so the following three equations are satisfied:

$$
\int_{t} \vec{e} \cdot \vec{e} \mathrm{~d} A=|t|, \quad \int_{t} \vec{f} \cdot \vec{f} \mathrm{~d} A=|t|, \quad \text { and } \quad \int_{t} \vec{e} \cdot \vec{f} \mathrm{~d} A=0
$$

It is clear that if a matrix $X_{t}$, representing a discrete inner product with respect to the standard basis $\left\{\hat{u}^{1}, \hat{u}^{2}, \hat{u}^{3}\right\}$, is to be exact on projections (onto $\mathcal{E}_{t}$ ) of constant vector fields (in $\mathbb{R}^{2}$ ) the following three equations must also be satisfied:

$$
[\hat{e}, \hat{e}]_{X_{t}}=|t|, \quad[\hat{f}, \hat{f}]_{X_{t}}=|t|, \quad \text { and } \quad[\hat{e}, \hat{f}]_{X_{t}}=0 .
$$

In what follows we alternately view the matrix $X_{t}$ as both a bilinear operator (form), $X_{t}: \mathcal{E}_{t} \times \mathcal{E}_{t} \longrightarrow \mathbb{R}$, and as a linear operator, $X_{t}: \mathcal{E}_{t} \longrightarrow \mathcal{E}_{t}$. The matrices described below that correspond to the various different 
methods of extending the vector $\hat{u}$ to $\vec{u}_{h}$ throughout the triangle $t$ will be studied in detail in their roles as both linear and bilinear operators.

\subsection{The matrix representations}

We denote by $\widetilde{W}_{t}$ the matrix that represents the mimetic inner product. This inner product results from the method of extending $\hat{u} \in \mathcal{E}_{t}$ to a piecewise (over subregions) constant vector field $\vec{u}_{h}$ in $t$ that is described in Section 2.3. Recall that the three subregions in the triangle $t$ (each of which is associated with a node) have areas (or nodal weights) $\left\{V_{1}, V_{2}, V_{3}\right\}$. The matrix that represents this inner product on $\mathcal{E}_{t}$ takes the form:

$$
\widetilde{W}_{t}=\left[\begin{array}{ccc}
\frac{V_{2}}{\sin ^{2} \phi_{2}}+\frac{V_{3}}{\sin ^{2} \phi_{3}} & \frac{V_{3} \cos \phi_{3}}{\sin ^{2} \phi_{3}} & \frac{V_{2} \cos \phi_{2}}{\sin ^{2} \phi_{2}} \\
\frac{V_{3} \cos \phi_{3}}{\sin ^{2} \phi_{3}} & \frac{V_{1}}{\sin ^{2} \phi_{1}}+\frac{V_{3}}{\sin ^{2} \phi_{3}} & \frac{V_{1} \cos \phi_{1}}{\sin ^{2} \phi_{1}} \\
\frac{V_{2} \cos \phi_{2}}{\sin ^{2} \phi_{2}} & \frac{V_{1} \cos \phi_{1}}{\sin ^{2} \phi_{1}} & \frac{V_{1}}{\sin ^{2} \phi_{1}}+\frac{V_{2}}{\sin ^{2} \phi_{2}}
\end{array}\right]
$$

Note that this matrix is not diagonal but that the $e \times e$ matrix $\widetilde{W}$ is sparse. In fact each row has at most five non-zero entries. However, the inverse of the matrix will not be sparse and the consequences of this are addressed in the final section.

Similarly, the matrix representation of the Raviart-Thomas/Nedelec inner product is not diagonal but is sparse.

To represent the matrix $W_{t}$ for the covolume method we introduce the following additional notation. Recall that the length of each edge $\sigma_{i}$ is denoted $h_{i}$ and the length of each coedge $\sigma_{i}^{\prime}$ is $h_{i}^{\prime}$. The length of the perpendicular bisector connecting the midpoint $\vec{x}_{i}$ of the edge to the circumcenter $\vec{c}$ of the triangle is denoted $h_{i}^{*}$. It is important to note that if the circumcenter lies outside the triangle (across an edge $\sigma_{i}$ ) then $h_{i}^{*}$ will be negative. In this case, however, the other two lengths $h^{*}$ in the triangle will be positive. See Figure 6 .

The matrix $W_{t}$ which represents the discrete inner product on $\mathcal{E}_{t} \subset \mathcal{E}$ with respect to the standard basis $\left\{\hat{u}^{1}, \hat{u}^{2}, \hat{u}^{3}\right\}$ is the following diagonal matrix:

$$
W_{t}=\left[\begin{array}{ccc}
h_{1} h_{1}^{*} & 0 & 0 \\
0 & h_{2} h_{2}^{*} & 0 \\
0 & 0 & h_{3} h_{3}^{*}
\end{array}\right]
$$

To ensure that the $e \times e$ matrix $W$, representing the covolume inner product on $\mathcal{E}$, is positive definite it is necessary that the triangulation be a Delaunay triangulation. In a Delaunay triangulation, while some of the $h_{i}^{*} s$ can be negative, the length $h_{i}^{\prime}$, which is the sum of two $h^{*}$ s never is. However, the restricted $3 \times 3$ matrix, $W_{t}$, need not be positive definite and therefore may not be a true inner product on $\mathbb{R}^{3}$. We are thus using the term "discrete inner product" on the space $\mathcal{E}_{t}$ to describe any bilinear form that approximates the $\left(L^{2}(t)\right)^{2}$ inner product. We show below that a semi-definite matrix can still yield accurate approximations to the $\left(L^{2}(t)\right)^{2}$ inner product.

\section{2. $\boldsymbol{L}^{2}$ accuracy}

While Section 2 allowed for any inner product on $\mathbb{R}^{e}$ to be admissible in this family of discretization methods, in practice only those inner products that satisfy equation (4.1) should be considered.

The mimetic method of extending $\hat{u}$ to $\vec{u}_{h}$ makes it clear that this discrete inner product satisfies equation (4.1). A constant vector field $\vec{u}$ in $t$ that is projected onto $\mathcal{E}_{t}$ will be reconstructed in each subregion of the triangle. The accuracy of the discrete mimetic inner product is independent of the choice of nodal subregions and their areas $\left\{V_{1}, V_{2}, V_{3}\right\}$.

Similarly, the Raviart-Thomas/Nedelec inner product will reconstruct a constant vector field projected onto $\mathcal{E}_{t}$. 


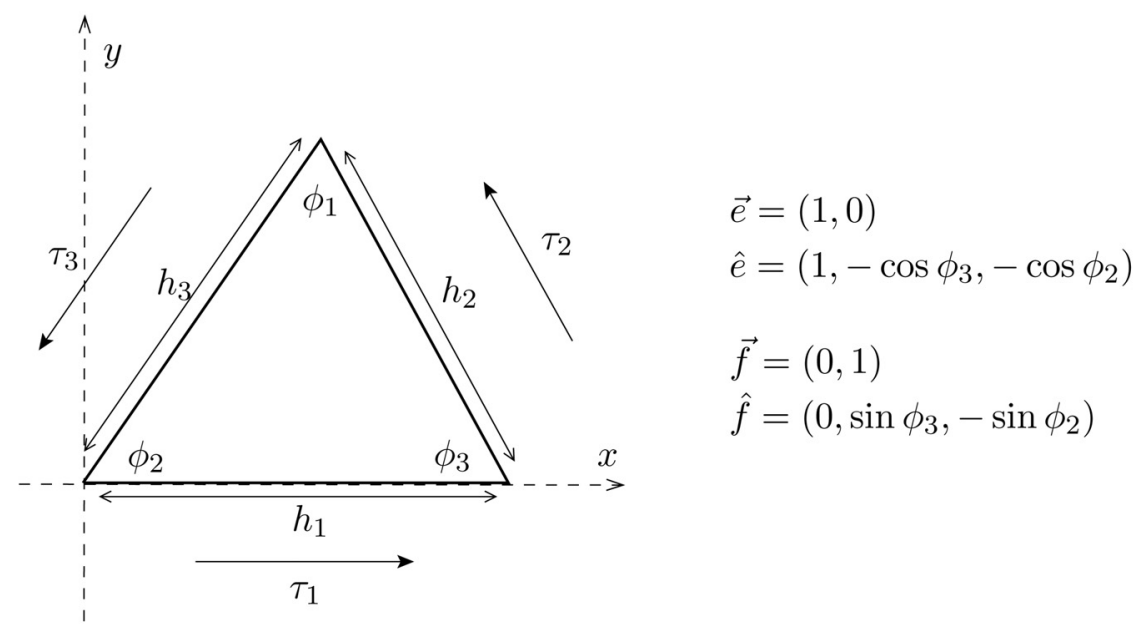

FiguRE 5. Triangle notation and alignment.
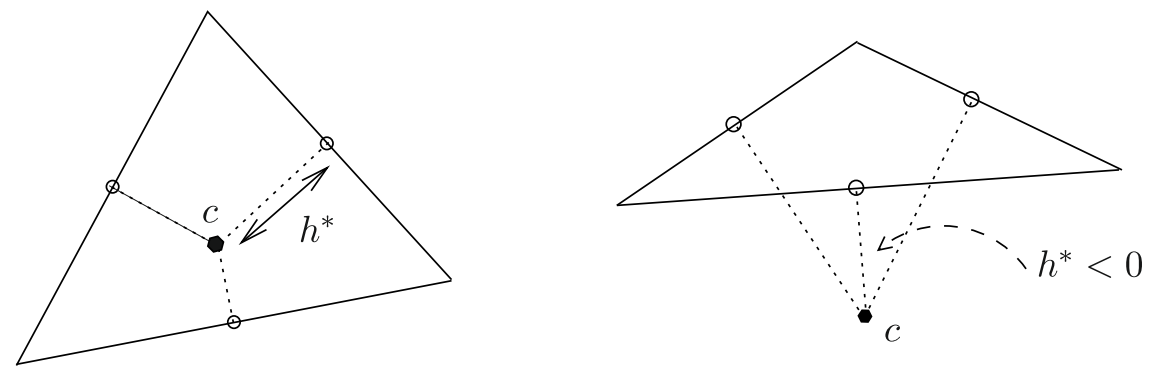

FiguRE 6. Signed $h^{*}$.

The covolume inner product is unique in that it is the only inner product on $\mathcal{E}$ with diagonal matrix $W$ that approximates the $L^{2}$ inner product exactly on projections of constant vector fields.

Theorem 4.1. The covolume inner product $W_{t}$ is the unique inner product on $\mathcal{E}_{t}$ that satisfies both of the following conditions:

(1) It is diagonal with respect to the standard basis $\left\{\hat{u}^{1}, \hat{u}^{2}, \hat{u}^{3}\right\}$.

(2) It satisfies equation (4.1).

Proof. Without loss of generality we consider the triangle from Figure 5 . We first show that there is only one $3 \times 3$ diagonal matrix $Q$ that satisfies equation (4.1) and then show that the covolume inner product $W_{t}$ is equal to $Q$.

Let $Q=\left[\begin{array}{ccc}q_{1} & 0 & 0 \\ 0 & q_{2} & 0 \\ 0 & 0 & q_{3}\end{array}\right]$.

To satisfy equation (4.1) we must have

$$
\hat{e} \cdot Q \hat{e}=|t|, \quad \hat{f} \cdot Q \hat{f}=|t|, \quad \text { and } \quad \hat{e} \cdot Q \hat{f}=0
$$


which we write as the following system of equations:

$$
\left[\begin{array}{ccc}
0 & -\sin \phi_{3} \cos \phi_{3} & \sin \phi_{2} \cos \phi_{2} \\
1 & \cos ^{2} \phi_{3} & \cos ^{2} \phi_{2} \\
0 & \sin ^{2} \phi_{3} & \sin ^{2} \phi_{2}
\end{array}\right]\left[\begin{array}{c}
q_{1} \\
q_{2} \\
q_{3}
\end{array}\right]=\left[\begin{array}{c}
0 \\
|t| \\
|t|
\end{array}\right]
$$

The determinant of this matrix is easily shown to be $D=\sin \phi_{1} \sin \phi_{2} \sin \phi_{3}$ which can not be zero and therefore the system has a unique solution $\left(q_{1}, q_{2}, q_{3}\right)$.

We now show that $q_{i}=h_{i} h_{i}^{*}$ for $i=1,2$, and 3 , by showing that the system of equations above is satisfied.

The necessary calculations follow from various trigonometric identities. The third equation is shown here:

$$
\begin{aligned}
h_{2} h_{2}^{*} \sin ^{2} \phi_{3}+h_{3} h_{3}^{*} \sin ^{2} \phi_{2} & =h_{3} \sin \phi_{2}\left(h_{2}^{*} \sin \phi_{3}+h_{3}^{*} \sin \phi_{2}\right) \\
& =\frac{1}{2} h_{3} \sin \phi_{2}\left(h_{3} \cos \phi_{2}+h_{2} \cos \phi_{3}\right) \\
& =\frac{1}{2} h_{1} h_{3} \sin \phi_{2} \\
& =|t| .
\end{aligned}
$$

The other two equations follow similarly. Thus $W_{t}=Q$ and the theorem is proved.

The covolume method has been applied to many systems of partial differential equations and convergence analysis has been extensive. In this analysis the behavior of the matrix $X_{t}$ as a linear operator is important. Therefore the class of matrices that agree with the covolume inner product matrix, $W_{t}$, as linear operators on the two-dimensional subspace $\hat{h}^{\perp} \in \mathcal{E}_{t}$ is of particular interest. Let

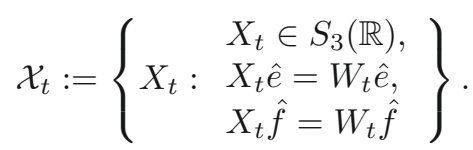

The conditions on $\mathcal{X}_{t}$ imply that every member $X_{t}$ also satisfies (4.1) and therefore every member of this class approximates the $\left(L^{2}(t)\right)^{2}$ inner product on constant vector fields exactly.

As an alternative way to represent the members of $\mathcal{X}$ we consider particular generalized eigenvectors for these matrices. Consider the vector $\hat{q}:=\left(\frac{1}{h_{1}^{*}}, \frac{1}{h_{2}^{*}}, \frac{1}{h_{3}^{*}}\right)$. It is clear that $\hat{q}=W_{t}^{-1} \hat{h}$.

From the orthogonality of $\hat{e}$ and $\hat{h}$ and also that of $\hat{f}$ and $\hat{h}$, we have

$$
[\hat{e}, \hat{q}]_{W_{t}}=0 \text { and }[\hat{f}, \hat{q}]_{W_{t}}=0
$$

Thus the vectors $\hat{e}, \hat{f}$, and $\hat{q}$ form a $W_{t}$-orthogonal basis for $\mathcal{E}_{t}$.

This orthogonality transfers to the entire set $\mathcal{X}$. Because $\hat{e}, \hat{f}$, and $\hat{q}$ are $X_{t}$-orthogonal for every $X_{t} \in \mathcal{X}_{t}$ we can write $X \hat{q}=\lambda W_{t} \hat{q}$ for some $\lambda \in \mathbb{R}$. Therefore, every matrix in $\mathcal{X}$ can be expressed in the following manner depending only on $\lambda \in \mathbb{R}$.

Let $E$ and $\Lambda$ be defined as

$$
E:=\left(\begin{array}{ccc} 
& & \\
\hat{e}^{T} & \hat{f}^{T} & \hat{q}^{T}
\end{array}\right), \quad \Lambda:=\left(\begin{array}{ccc}
1 & 0 & 0 \\
0 & 1 & 0 \\
0 & 0 & \lambda
\end{array}\right) .
$$

Then

$$
X_{t} E=E \Lambda W_{t}
$$

and there is a one-to-one correspondence between $\left\{X_{t} \in \mathcal{X}_{t}\right\} \longleftrightarrow\{\lambda \in \mathbb{R}\}$. 
Theorem 4.2. The mimetic inner product matrix $\widetilde{W}_{t}$ with nodal weights $V_{i}:=|t| \frac{\tan \phi_{i}}{\sum_{k=1}^{3} \tan \phi_{k}}$, is in the class $\mathcal{X}_{t}$ and the generalized eigenvalue associated with $\hat{q}=\left(\frac{1}{h_{1}^{*}}, \frac{1}{h_{2}^{*}}, \frac{1}{h_{3}^{*}}\right)$ is $\lambda=\sum_{k} \frac{h_{k}}{h_{k}^{*}}$.

Proof. We need to show that $\widetilde{W}_{t} \hat{e}=W_{t} \hat{e}$ and $\widetilde{W}_{t} \hat{f}=W_{t} \hat{f}$. We will show in detail that $\left(\widetilde{W}_{t} \hat{e}\right)_{1}=\left(W_{t} \hat{e}\right)_{1}$. The other equations and the generalized eigenvalue calculation follow similarly.

Using the trigonometric identities $2 \sum_{k} \tan \phi_{k}=\sum_{k} \frac{h_{k}}{h_{k}^{*}}, h_{1}=2\left(h_{2}^{*} \sin \phi_{3}+h_{3}^{*} \sin \phi_{2}\right), h_{2}^{*}=\frac{h_{3} \cos \phi_{2}}{2 \sin \phi_{3}}$, and the easily derived $\frac{h_{1}}{h_{1}^{*}}+\frac{h_{2}}{h_{2}^{*}}=\frac{h_{3}}{h_{1}^{*} \cos \phi_{2}}$ the matrix calculation reduces to

$$
\begin{aligned}
\left(\widetilde{W}_{t} \hat{e}\right)_{1}= & \frac{|t|}{\sum_{k} \tan \phi_{k}}\left(\frac{\tan \phi_{2}}{\sin ^{2} \phi_{2}}+\frac{\tan \phi_{3}}{\sin ^{2} \phi_{3}}-\frac{\tan \phi_{3} \cos \phi_{3}}{\sin ^{2} \phi_{3}}-\frac{\tan \phi_{2} \cos \phi_{2}}{\sin ^{2} \phi_{2}}\right) \\
& =\frac{|t|}{\sum_{k} \tan \phi_{k}}\left(\frac{1}{\sin \phi_{2} \cos \phi_{2}}+\frac{1}{\sin \phi_{3} \cos \phi_{3}}-\frac{\cos \phi_{3}}{\sin \phi_{3}}-\frac{\cos \phi_{2}}{\sin \phi_{2}}\right) \\
& =\frac{|t|}{\sum_{k} \tan \phi_{k}}\left(\frac{1}{\sin \phi_{2} \cos \phi_{2}}+\frac{1}{\sin \phi_{3} \cos \phi_{3}}-\frac{\cos \phi_{3}}{\sin \phi_{3}}+\frac{h_{3}\left(h_{2} \cos \phi_{3}-h_{1}\right)}{h_{3} h_{2} \sin \phi_{3}}\right) \\
& =\frac{|t|}{\sum_{k} \tan \phi_{k}}\left(\frac{1}{\sin \phi_{2} \cos \phi_{2}}+\frac{1}{\sin \phi_{3} \cos \phi_{3}}-\frac{h_{1}}{h_{2} \sin \phi_{3}}\right) \\
& =h_{1} h_{1}^{*} \frac{|t|}{\sum_{k} \tan \phi_{k}}\left(\frac{1}{h_{1} h_{1}^{*} \sin \phi_{2} \cos \phi_{2}}+\frac{1}{h_{1} h_{1}^{*} \sin \phi_{3} \cos \phi_{3}}-\frac{1}{h_{1}^{*} h_{2} \sin \phi_{3}}\right) \\
& =h_{1} h_{1}^{*} \frac{|t|}{\sum_{k} \tan \phi_{k}}\left(\frac{1}{h_{1} h_{1}^{*} \sin \phi_{2} \cos \phi_{2}} \frac{h_{3}}{h_{3}}+\frac{1}{h_{1} h_{1}^{*} \sin \phi_{3} \cos \phi_{3}} \frac{h_{2}}{h_{2}}-\frac{1}{h_{1}^{*} h_{2} \sin \phi_{3}} \frac{h_{1}}{h_{1}}\right) \\
& =h_{1} h_{1}^{*} \frac{1}{2 \sum_{k} \tan \phi_{k}}\left(\frac{h_{3}}{h_{1}^{*} \cos \phi_{2}}+\frac{h_{2}}{h_{1}^{*} \cos \phi_{3}}-\frac{h_{1}}{h_{1}^{*}}\right) \\
& =h_{1} h_{1}^{*} \frac{1}{2 \sum_{k} \tan \phi_{k}}\left(\frac{h_{1}}{h_{1}^{*}}+\frac{h_{2}}{h_{2}^{*}}+\frac{h_{1}}{h_{1}^{*}}+\frac{h_{3}}{h_{3}^{*}}-\frac{h_{1}}{h_{1}^{*}}\right) \\
& =h_{1} h_{1}^{*} \\
& =\left(W_{t} \hat{e}\right)_{1} .
\end{aligned}
$$

Corollary 4.1. For a triangle $t$ the nodal weights $\left\{V_{1}, V_{2}, V_{3}\right\}$ defined above satisfy $V_{1}+V_{2}=h_{3} h_{3}^{*}, V_{2}+V_{3}=$ $h_{1} h_{1}^{*}$, and $V_{1}+V_{3}=h_{2} h_{2}^{*}$.

Proof. Use identities and calculations similar to the above.

\subsection{The derived divergence stencil}

An important feature of the covolume inner product matrix $W$ is that it is diagonal. The dual operators $K^{*}$ and $C^{*}$ were derived using $W^{-1}$. Thus, unlike the mimetic and Raviart-Thomas/Nedelec matrices, the covolume matrix $W$ yields dual operators which all have local stencils. This has two important consequences. One is a computational advantage: in solving the systems of equations in which these operators arise, the inversion of matrices is computationally time-consuming. Secondly, the dual discrete divergence operator has an interpretation as an actual approximation of the divergence of a vector field over a covolume region.

Recall that the dual divergence operator has the form $D^{*}=-N^{-1} G^{T} W$ and that in the covolume method the nodal weights in the matrix inner product on $\mathcal{N}$ are $\left|t_{k}^{\prime}\right|$. The stencil of the dual divergence operator is shown in Figure 7.

In our description of the class of schemes that fit into the exact sequence framework, the choice of inner product determined the dual discrete divergence operator. It is possible to go backwards: choosing a discrete divergence operator that acts on $\mathcal{E}_{0}$ and results in elements of $\mathcal{N}_{0}$ creates a discrete inner product on the space $\mathcal{E}$. 


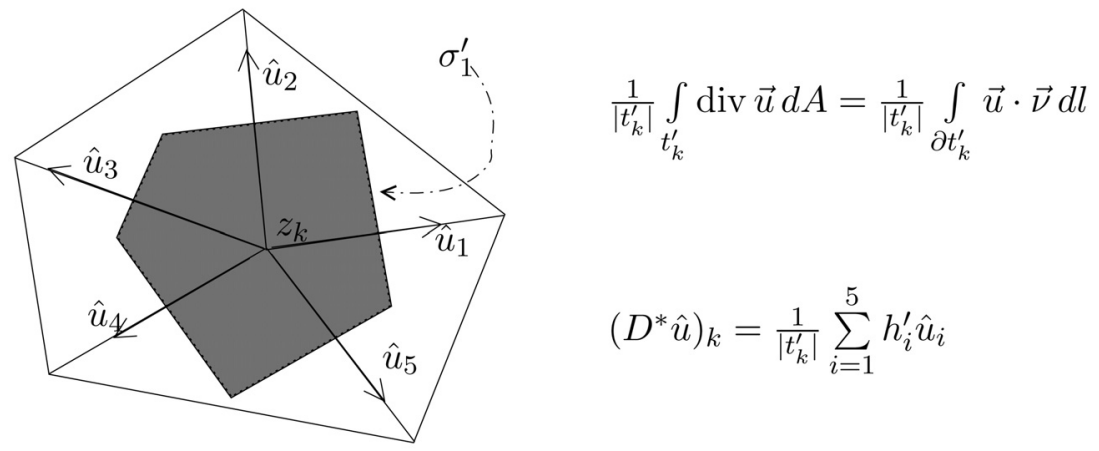

FiguRE 7. Covolume divergence operator stencil.

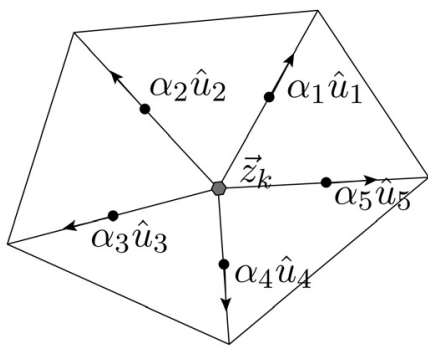

$$
\left(D^{*} \hat{u}\right)_{k}=\frac{1}{N_{k}} \sum_{i=1}^{5} \alpha_{i} \hat{u}_{i}
$$

FiguRE 8. General diagonal matrix induced divergence operator stencil.

Any choice of diagonal matrix $Q$ representing a discrete inner product on $\mathcal{E}$ creates a dual divergence operator $D^{*}=-N^{-1} G^{T} Q$ that has a local stencil. Similarly, if $\left(D^{*} \hat{u}\right)_{k}:=\frac{1}{N_{k}} \sum_{i=1}^{5} \alpha_{i} \hat{u}_{i}$ for some positive set of $\alpha_{i} s$ then the discrete inner product matrix $Q$ on $\mathcal{E}$ must be diagonal and satisfy $Q_{i i}=h_{i} \alpha_{i}$. See Figure 8 .

One benefit of the local stencil is the ability to analyze the accuracy of the discrete divergence operator. From Figures 7 and 8 we see that if $\alpha_{i}=h_{i}^{\prime}$ for $i=1, \ldots, 5$, then for a constant vector field $\vec{u}$ and its projection $\hat{u}$, the dual divergence $D^{*}$ satisfies

$$
\left(D^{*} \hat{u}\right)_{k}=\frac{1}{\left|N_{k}\right|} \int_{N_{k}} \operatorname{div} \vec{u} \mathrm{~d} A=0
$$

where $N_{k}=t_{k}^{\prime}$. We refer to an operator that satisfies this property (for any choice of nodal region $N_{k}$ ) as div-accurate.

This notion immediately raises two questions: for what other choices of $\left\{\alpha_{i}\right\}_{i=1}^{5}$ is this the case? And what nodal regions (i.e. what choice of discrete inner product matrix $N$ on $\mathcal{N}$ ) can be associated with these $\alpha_{i}$ edge weights so that the dual discrete divergence is div-accurate? We categorize the $\alpha_{i} \mathrm{~s}$ in the following theorem. Consider Figure 9.

Theorem 4.3. Given $n$ points in $\mathbb{R}^{2},\left\{\vec{x}_{i}\right\}_{i=1}^{n}$, and positive scalar values $\left\{\alpha_{i}\right\}_{i=1}^{n}$, for $i=1, \ldots, n$ let $\vec{x}_{i}=\left(x_{i}, y_{i}\right)$ and define $\vec{n}_{i}=\frac{1}{\left\|\vec{x}_{i}\right\|}\left(x_{i}, y_{i}\right)$ and $\vec{\tau}_{i}=\frac{1}{\left\|\vec{x}_{i}\right\|}\left(y_{i},-x_{i}\right)$. Given $\vec{u} \equiv\left(c_{1}, c_{2}\right)$ define $u_{i}=\vec{u} \cdot \vec{n}_{i}$ for $i=1, \ldots, n$. Then $\sum_{i=1}^{n} \alpha_{i} u_{i}=0$ for all constants $c_{1}$ and $c_{2}$ if and only if there exists a n-sided polygon $P$ with sides $\sigma_{i}^{\prime}$ perpendicular to $\vec{x}_{i}$ and of length $\alpha_{i}$ for $i=1, \ldots, n$. 


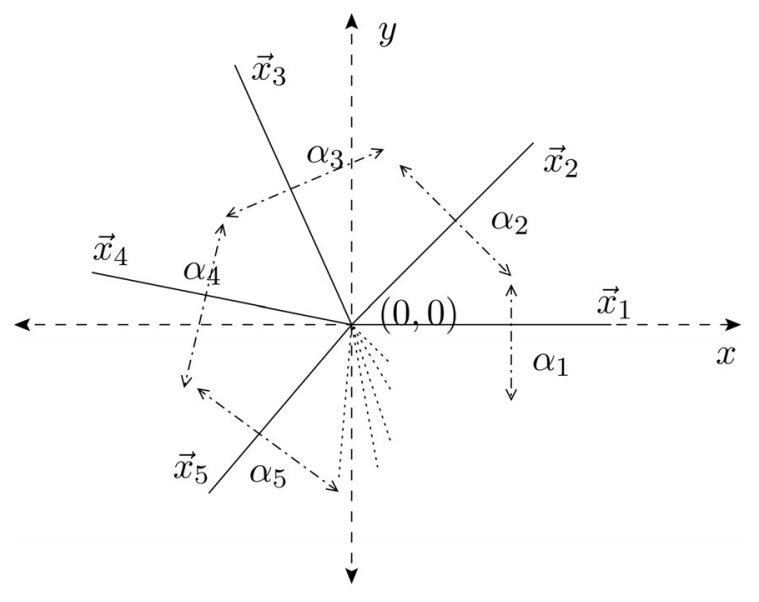

Figure 9. Theorem 4.1 diagram.

Proof. $(\Longleftarrow)$ The vector field $\vec{u}$ is constant over the polygon $P$ so that $\operatorname{div} \vec{u}=0$. Therefore

$$
0=\int_{P} \operatorname{div} \vec{u} \mathrm{~d} A=\int_{\partial P} \vec{u} \cdot \vec{n} \mathrm{~d} s=\sum_{i=1}^{n} \int_{\sigma_{i}^{\prime}} u_{i} \mathrm{~d} s=\sum_{i=1}^{n} \alpha_{i} u_{i} .
$$

$(\Longrightarrow)$ Since $\vec{x}_{i} \cdot \vec{\tau}_{i}=0$ for all $i$ it suffices to show that the sides $\sigma_{i}^{\prime}:=\alpha_{i} \vec{\tau}_{i}$ create a polygon. In turn, that reduces to showing that $\sum_{i=1}^{n} \alpha_{i} \vec{\tau}_{i}=\overrightarrow{0}$.

Let $\vec{e}=(1,0)$ and $\vec{f}=(0,1)$. By the hypotheses $\sum_{i=1}^{n} \alpha_{i} e_{i}=0$ and $\sum_{i=1}^{n} \alpha_{i} f_{i}=0$. Thus:

$$
\begin{aligned}
\sum_{i=1}^{n} \alpha_{i} \vec{\tau}_{i} & =\sum_{i=1}^{n}\left(\alpha_{i} \frac{y_{i}}{\left\|x_{i}\right\|},-\alpha_{i} \frac{x_{i}}{\left\|x_{i}\right\|}\right) \\
& =\left(\sum_{i=1}^{n} \alpha_{i} \frac{y_{i}}{\left\|x_{i}\right\|},-\sum_{i=1}^{n} \alpha_{i} \frac{x_{i}}{\left\|x_{i}\right\|}\right) \\
& =\left(\sum_{i=1}^{n} \alpha_{i} \vec{e} \cdot \vec{n}_{i},-\sum_{i=1}^{n} \alpha_{i} \vec{f} \cdot \vec{n}_{i}\right) \\
& =(0,0) .
\end{aligned}
$$

Thus we can only be assured div-accuracy of $D^{*}=-N^{-1} G^{T} Q$ (with diagonal matrix $Q$ ) if the weights in $Q$ correspond to the lengths of the sides of a polygon and these sides are perpendicular to the edges of the mesh. Any other choice of regions will not have accurate divergence stencils. The additional requirement that the nodal regions cover the entire domain $\Omega_{h}$ suggests that the covolume matrix $W_{t}$ is unique among diagonal matrices (representing inner products on $\mathcal{E}_{t}$ ) in yielding a div-accurate dual divergence operator.

\section{Conclusion}

In the partial differential equations that these methods have been applied to, the choice of inner product does not affect the existence and uniqueness of solutions to the systems of equations. Thus it is the computability and convergence analysis that varies. 
The choice of which inner product to choose must mainly depend on the mesh. For Delaunay triangulations, the benefits of the covolume's diagonal matrix inner product are clear. For non-Delaunay triangulations, where the covolume method is not applicable, it is possible that an inner product from the set $\mathcal{X}$ may be most suitable for convergence analysis. For more general meshes, and in particular quadrilateral meshes, the mimetic method appears most appropriate.

This construction of discrete spaces and discrete operators, and the existence of exact sequences can be extended to higher order discretizations and higher dimensions. For higher order discretizations in this framework see [18] and for a differential forms viewpoint of this construction see $[3,14]$.

\section{REFERENCES}

[1] D.N. Arnold, Differential complexes and numerical stability, in Proceedings of the International Congress of Mathematicians, Vol. I, Higher Ed. Press, Beijing (2002) 137-157.

[2] M. Berndt, K. Lipnikov, D. Moulton and M. Shashkov, Convergence of mimetic finite difference discretizations of the diffusion equation. East-West J. Numer. Math 9 (2001) 253-316.

[3] P. Bochev and J.M. Hyman, Principles of mimetic discretizations of differential operators, in Compatible Spatial Discretizations, D. Arnold, P. Bochev, R. Lehoucq, R. Nicolaides and M. Shashkov Eds., IMA Volumes in Mathematics and its Applications 142, Springer, New York (2006).

[4] A. Bossavit, Generating whitney forms of polynomial degree one and higher. IEEE Trans. Magn. 38 (2002) 341-344.

[5] R. Hiptmair, Canonical construction of finite elements. Math. Comp. 68 (1999) 1325-1346.

[6] A. Hirani, Discrete Exterior Calculus. Ph.D. thesis, California Institute of Technology, USA (2003).

[7] J.M. Hyman and M. Shashkov, The adjoint operators for the natural discretizations for the divergence, gradient, and curl on logically rectangular grids. IMACS J. Appl. Num. Math. 25 (1997) 1-30.

[8] J.M. Hyman and M. Shashkov, Natural discretizations for the divergence, gradient, and curl on logically rectangular grids. Comput. Math. Appl. 33 (1997) 81-104.

[9] J.M. Hyman and M. Shashkov, Mimetic discretizations for Maxwell's equations. J. Comp. Phys. 151 (1999) 881-909.

[10] J.M. Hyman and M. Shashkov, The orthogonal decomposition theorems for mimetic finite difference methods. SIAM J. Numer. Anal. 36 (1999) 788-818.

[11] J.C. Nedelec, Mixed finite elements in $\mathbb{R}^{3}$. Numer. Math. 35 (1980) 315-341.

[12] J.C. Nedelec, A new family of mixed finite elements in $\mathbb{R}^{3}$. Numer. Math. 50 (1986) 57-81.

[13] R.A. Nicolaides, Direct discretization of planar div-curl problems. SIAM J. Numer. Anal. 29 (1992) 32-56.

[14] R. Nicolaides and K. Trapp, Covolume discretizations of differential forms, in Compatible Spatial Discretizations, D. Arnold, P. Bochev, R. Lehoucq, R. Nicolaides and M. Shashkov Eds., IMA Volumes in Mathematics and its Applications 142, Springer, New York (2006).

[15] R.A. Nicolaides and D.Q. Wang, Convergence analysis of a covolume scheme for Maxwell's equations in three dimensions. Math. Comp. 67 (1998) 947-963.

[16] R.A. Nicolaides and X. Wu, Covolume solutions of three-dimensional div-curl equations. SIAM J. Numer. Anal. 34 (1997) $2195-2203$.

[17] P.A. Raviart and J.M. Thomas, A mixed finite elemnt method for second order elliptic problems, in Springer Lecture Notes in Mathematics 606, Springer-Verlag (1977) 292-315.

[18] K. Trapp, A Class of Compatible Discretizations with Applications to Div-Curl Systems. Ph.D. thesis, Carnegie Mellon University, USA (2004). 